\title{
Massive star and star cluster formation
}

\author{
Jonathan C. Tan $^{1}$ \\ ${ }^{1}$ Department of Astronomy, University of Florida, Gainesville, FL 32611, USA \\ email: jt @ astro.ufl.edu
}

\begin{abstract}
I review the status of massive star formation theories: accretion from collapsing, massive, turbulent cores; competitive accretion; and stellar collisions. I conclude the observational and theoretical evidence favors the first of these models. I then discuss: the initial conditions of star cluster formation as traced by infrared dark clouds; the cluster formation timescale; and comparison of the initial cluster mass function in different galactic environments.
\end{abstract}

\section{Introduction}

Massive stars and star clusters form together as part of a single unified process. All locally-observed massive stars appear to form in star clusters (de Wit et al. 2005), particularly in rich star clusters (Massi, Testi \& Vanzi 2006). Star clusters make a significant, perhaps dominant, contribution to the total star formation rate of galaxies (Lada \& Lada 2003; Fall, Chandar, \& Whitmore 2005), so to understand global star formation properties of galaxies (e.g. Kennicutt 1998), one must understand star cluster formation.

\section{Massive star formation}

There is still some debate about how massive stars form. Do they form from the global collapse of a massive, initially starless gas core, in which a central protostar or binary grows from low to high mass by accretion from a disk (e.g. McKee \& Tan 2003)? This is a scaled-up version of the standard model of low-mass star formation (Shu, Adams, \& Lizano 1987). Or do they form from favored low-mass protostellar seeds that accrete gas competitively, with the gas being bound to the protocluster potential but not at any stage in a spatially coherent bound core with a mass similar to that of the final massive star (e.g. Bonnell, Vine, \& Bate 2004). These latter models typically involve the global collapse of the protocluster gas over a timescale approximately equal to its free-fall time, so the growth of the massive star takes place on the same timescale as the formation of the entire cluster. It has been suggested that protostellar collisions may also be involved in the growth of massive stars (Bonnell, Bate, \& Zinnecker 1998; Bally \& Zinnecker 2005).

Evidence in support of the core model of massive star formation includes the fact that massive starless cores are observed and the mass function of these cores is similar to the stellar initial mass function (IMF) (Beuther \& Schilke 2004; Reid \& Wilson 2006). Massive cores tend to have line widths that are much broader than thermal (Caselli \& Myers 1995), indicating that other forms of pressure support such as turbulent motions and magnetic fields are important. Indeed observed magnetic field strengths are close to the values needed to support the gas (Crutcher 2005). Known massive protostars tend to be embedded in dense gas cores with masses comparable to the stellar masses (e.g. Source I in the Orion Hot Core; W3 $\left(\mathrm{H}_{2} \mathrm{O}\right)$ ). Low-mass protostars, i.e. actively accreting stars, always have relatively massive accretion disks and outflows. A number of claims have been made for disks around massive protostars, although it is usually difficult to determine if these are rotationally supported structures (see Cesaroni et al. 2006 for a review). 
Powerful outflows from massive protostars with similar degrees of collimation to those from low-mass protostars have been seen (Beuther et al. 2002). The expected evolutionary scheme for high-mass star formation from cores has been reviewed in more detail by Beuther et al. (2006). Doty, van Dishoeck, \& Tan (2006) considered the chemical evolution of this model with particular application to observations of water abundance in hot cores. Kratter \& Matzner (2006) investigated the gravitational stability of massive protostellar accretion disks. Krumholz, McKee, \& Klein (2007) presented radiation-hydrodynamic simulations of massive star formation from a massive turbulent core.

Massive star formation models involving competitive accretion and stellar collisions face several observational and theoretical hurdles. Edgar \& Clarke (2004) showed that Bondi-Hoyle accretion becomes very inefficient for protostellar masses $\gtrsim 10 M_{\odot}$ because of radiation pressure on dust in the gas. This feedback has not been included in any of the simulations in which massive stars form by competitive accretion.

To overcome radiation pressure the accretion flow to a massive star must become optically thick, either in a dense core or disk, or in collisions of protostars. The collisional timescale is $t_{\text {coll }}=1.44 \times 10^{10}\left(n_{*} / 10^{4} \mathrm{pc}^{-3}\right)^{-1}\left(\sigma / 2 \mathrm{~km} \mathrm{~s}^{-1}\right)\left(r_{*} / 10 R_{\odot}\right)^{-1}\left(m_{*} / M_{\odot}\right)^{-1} \mathrm{yr}$ in the limit of strong gravitational focusing, where $\sigma$ is the $1 \mathrm{D}$ velocity dispersion and $r_{*}$ is the radius of the stellar collisional cross-section. For collisions to occur frequently enough to grow a massive protostar within $10^{6} \mathrm{yr}$ (massive zero age main sequence stars are observed) requires protostellar densities of at least $10^{6} \mathrm{pc}^{-3}$ and probably closer to $10^{8} \mathrm{pc}^{-3}$, whereas typical observed stellar densities around massive protostars are much smaller. For example from the Orion Nebula Cluster (ONC) x-ray observations of Garmire et al. (2000), Tan (2004) estimates a stellar density of about $10^{5} \mathrm{pc}^{-3}$ in the KL region. This result is not significantly changed by the deeper x-ray observations of Grosso et al. (2005). Hunter et al. (2006) find a density of sub-mm cores in the center of protoclusters in NGC 6334 of about $10^{4} \mathrm{pc}^{-3}$. From Fig. 1 and the data of Mueller et al. (2002) we see that typical mean densities of the central regions of Galactic protoclusters are $n_{\mathrm{H}} \simeq 2 \times 10^{5} \mathrm{~cm}^{-3}$, i.e. $7000 M_{\odot} \mathrm{pc}^{-3}$. If all this gas mass formed stars, stellar densities would be about $10^{4} \mathrm{pc}^{-3}$, given a typical IMF. The fiducial core that forms a massive star in the model of McKee \& Tan (2003) is also shown in Fig. 1, and has a mean density about one to ten times greater than this. Even if the core fragmented with $100 \%$ efficiency into low-mass stars the stellar density would be too low for efficient growth via stellar collisions. In fact numerical simulations show that fragmentation of the core into many stars is impeded by heat input from the forming central massive star (Krumholz 2006). The numerical simulations in which greater degrees of fragmentation are seen (e.g. Dobbs, Bonnell, \& Clark 2005) do not include this feedback. Magnetic pressure is also likely to be important for the support of cores more massive than the thermal Jeans mass, but this is also usually not included in simulations of massive star formation.

If collisions are relevant for massive star formation, but not low-mass star formation, then one might expect a change in the slope of the stellar IMF at the mass scale at which the collisional process becomes important. In fact the stellar IMF is reasonably well-fit by a power law from $\sim 1 M_{\odot}$ out to the highest observed masses (Massey 1998).

\section{Star cluster formation}

\subsection{The initial conditions for star cluster formation: infrared dark clouds}

We expect the initial conditions for star clusters to be the densest starless gas clouds. Such clouds reveal themselves by absorption of the Galactic diffuse infrared background and have become known as Infrared Dark Clouds (IRDCs) (Egan et al. 1998). 


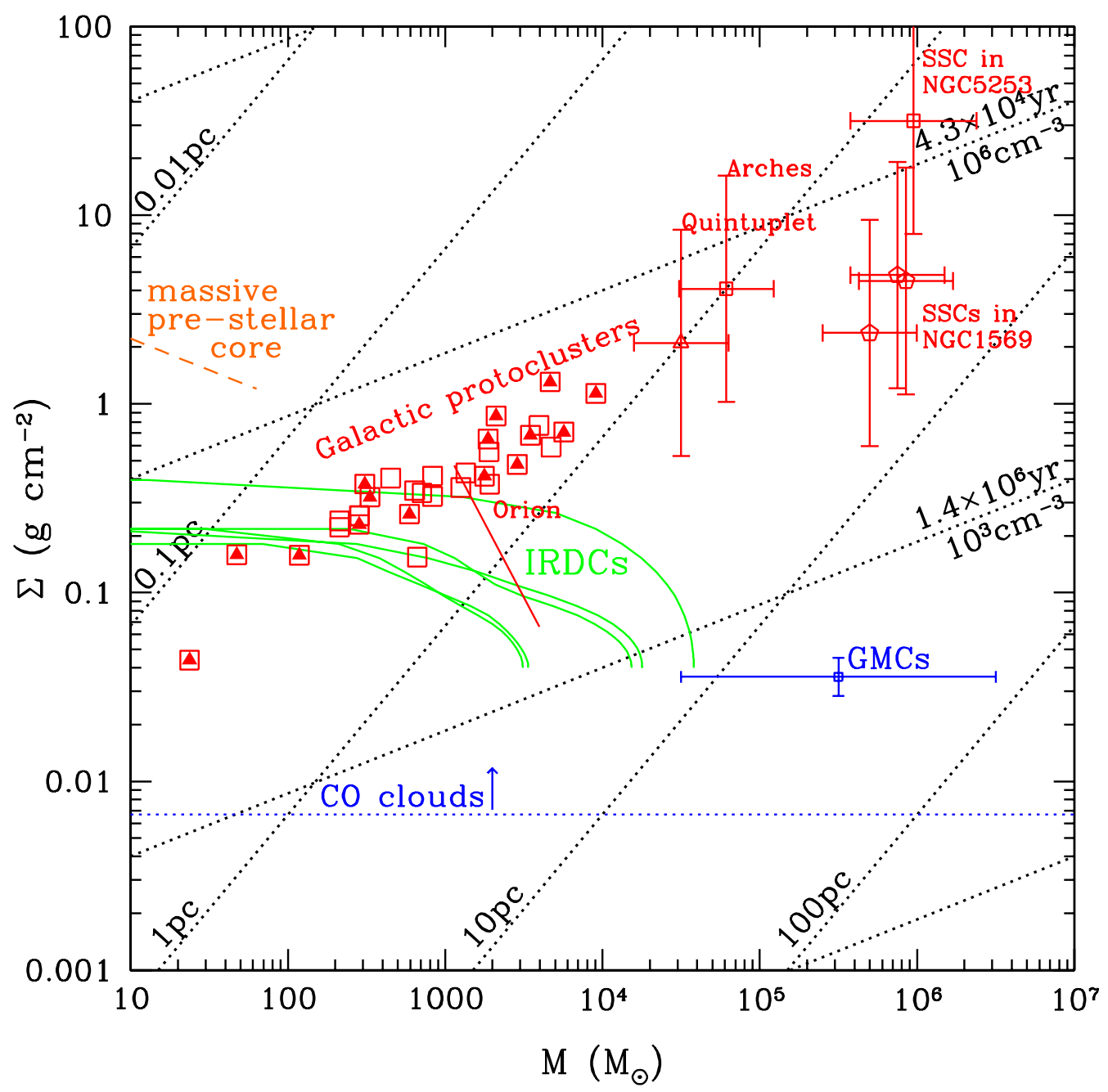

Figure 1. Surface density, $\Sigma \equiv M /\left(\pi R^{2}\right)$, versus mass, $M$, for star clusters and interstellar clouds. Contours of constant radius, $R$, and hydrogen number density, $n_{\mathrm{H}}$, or free-fall timescale, $t_{\mathrm{ff}}$, are shown with dotted lines. The minimum $\Sigma$ for CO clouds in the local Galactic FUV radiation field is shown, as are typical GMC parameters and the distributions $M(>\Sigma)$ of several IRDCs derived from extinction mapping (Butler et al., in prep.). Open squares are star-forming clumps (Mueller et al. 2002): a triangle indicates presence of an HII region. The solid straight line traces conditions from the inner to outer parts of the ONC, assuming equal mass in gas and stars. Several more massive clusters are also indicated. The fiducial massive core in the model of McKee \& Tan (2003) is shown by the dashed line.

One way to measure the physical properties of these clouds is through extinction mapping (Fig. 2). Assuming the diffuse Galactic infrared emission behind the cloud is similar to that around it and adopting an infrared extinction law and dust to gas ratio (Weingartner \& Draine 2001) allows the measurement of mass surface density, $\Sigma$. A kinematic distance can be measured from ${ }^{13} \mathrm{CO}$ line emission (Simon et al. 2001), and thus the physical size and mass of the cloud determined. The cumulative distributions of $M(>\Sigma)$, i.e. the mass that is at surface densities greater than or equal to a particular 


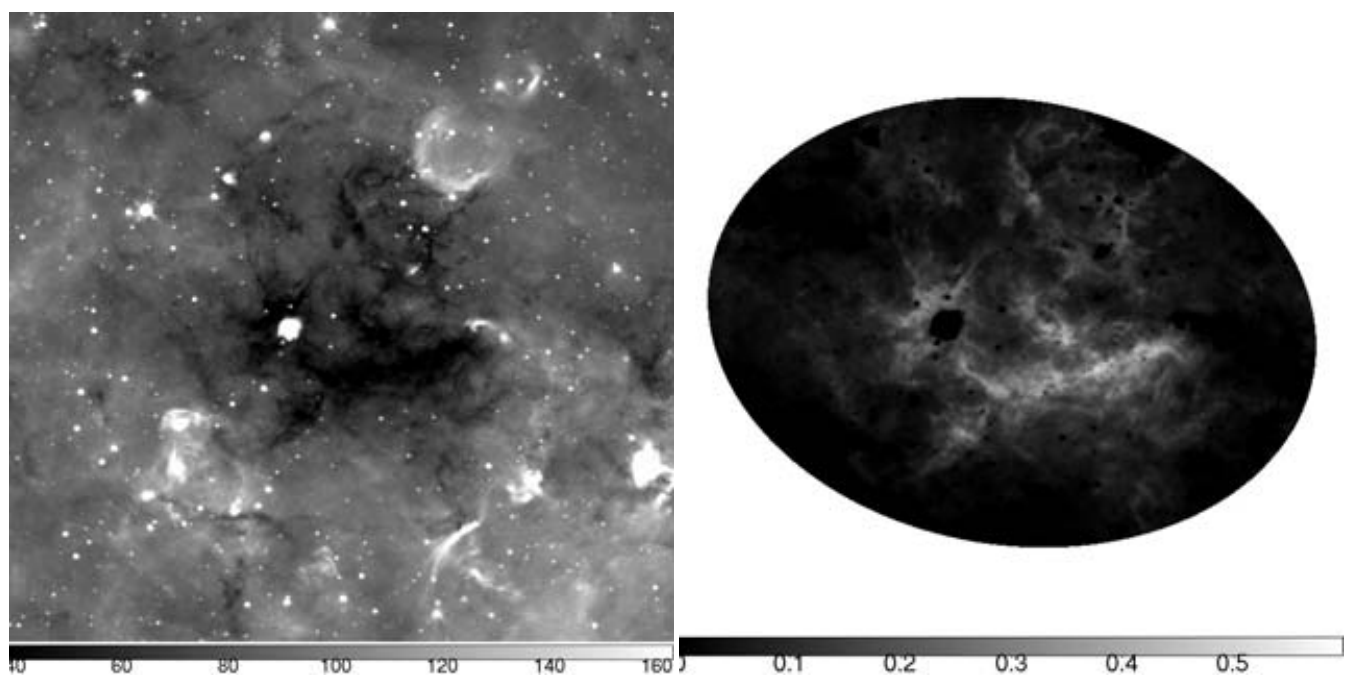

Figure 2. (a) Left: Example IRDC at $l=28.37, b=0.07$ and distance $4.9 \mathrm{kpc}$ observed by Spitzer at $8 \mu \mathrm{m}$. Image is $16.5^{\prime}$ across. (b) Right: $\Sigma$ map of the same cloud derived from extinction of the diffuse background (Butler et al., in prep). Intensity scale is in $\mathrm{g} \mathrm{cm}^{-2}$. Note the extinction mapping technique fails where there is a bright source in front of or in the cloud.

$\Sigma$, for five typical IRDCs have been measured by Butler, Tan, \& Hernandez, in prep and are shown in Fig. 1. The IRDCs span physical properties similar to those of embedded star clusters (Mueller et al. 2002), although with somewhat lower surface densities, and so are likely to be representative of the initial conditions of star cluster formation.

\subsection{The timescale for star cluster formation}

Some models of massive star and star cluster formation involve the global collapse of the protocluster in about one free-fall time (e.g. Bonnell, Vine \& Bate 2004), while other models that include feedback from the forming stars (e.g. Li \& Nakamura 2006) have star formation occurring more gradually over at least several free-fall times. Based on the results of numerical simulations, Krumholz \& McKee (2005) argued that supersonically turbulent gas forms stars at a slow rate of only a few percent of the total gas mass per dynamical or free-fall time. Tan, Krumholz, \& McKee (2006) extended this analysis to spherical clumps and argued that those clumps that eventually turn a high $(\gtrsim 30 \%)$ fraction of their mass into stars must do so over at least several $(\gtrsim 7)$ free-fall times.

Tan et al. (2006) also summarized the observational evidence in support of slow, quasiequilibrium star cluster formation: (1) The morphologies of CS gas clumps are round (Shirley et al. 2003); (2) the spatial distributions of stars in embedded, rich, i.e. high-starformation-efficiency, star clusters show relatively little substructure; (3) the momentum flux from the combined outflows from protostars in forming clusters is relatively small; (4) the age spreads of stars in rich star clusters are much greater than their current free-fall times; (5) in the ONC a dynamical ejection event associated with the cluster has been dated at 2.5 Myr (Hoogerwerf, de Bruijne, \& de Zeeuw 2001), which is much longer than the free-fall time of the present cluster.

Note it is the central, high-star-formation-efficiency region of the cluster where we propose that star formation takes place over several to many free-fall times. These regions have short free-fall times (see Fig. 1), $10^{5} \mathrm{yr}$. The outskirts of the cluster have much lower densities, longer free-fall times, and star formation here may occur over just one or two free-fall times, as proposed by Elmegreen (2000), before gas is disrupted by feedback 
from the newly-formed cluster. The global star formation efficiency here will be relatively low and the young stars will exhibit a greater degree of substructure.

It has been suggested that the star cluster formation process takes only one or two free-fall times when this time is referenced to the pre-cluster conditions at lower density, and that therefore star cluster formation can be regarded as being the result of dynamic collapse of a cloud and is not a quasi-equilibrium process (Hartmann \& Burkert 2006). This distinction is important because Krumholz, McKee, \& Klein (2005) showed that the process of star formation by competitive accretion cannot be important in virialized, equilibrium clouds. It requires sub-virial conditions associated with global collapse. Several arguments can be made against the global collapse picture: (1) the protocluster gas clouds appear to be approximately virialized (e.g. Shirley et al. 2003); (2) the final distributions of the distances of the stars from the cluster center should reflect the locations at which they formed or be even larger because of gas removal, yet we see newly formed rich star clusters with concentrated, dynamically-relaxed distributions. Huff \& Stahler (2006) found the star formation history of the ONC showed no dependence on the radial distance from the cluster center; (3) again in the ONC, the 2.5 Myr dynamical ejection event (Hoogerwerf et al. 2001) suggests that at this time the cluster was already in a state of high stellar density with a short free-fall time.

\subsection{The initial cluster mass function}

The initial cluster mass function (ICMF) is a fundamental property of the star cluster formation process. If there are external triggers, e.g. cloud collisions, supernova blast waves, that initiate star cluster formation, then these may influence the ICMF. It has been suggested that super star cluster formation may be favored in the low-shear environment of dwarf irregulars (Billett, Hunter, \& Elmegreen 2002).

To investigate whether the ICMF depends on galactic environment, Dowell, Buckalew, \& Tan (2007) used automated source selection from Sloan Digital Sky Survey (SDSS) data to measured the ICMF at masses $\gtrsim 3 \times 10^{4} M_{\odot}$ in 13 nearby $(\lesssim 10 \mathrm{Mpc})$ dwarf irregular galaxies, which tend to have relatively low metallicity and shear. Cluster ages, masses and reddening were determined by comparing Starburst99 models with the multicolor photometry. Completeness corrections were made, although these are relatively small for massive young clusters at these distances. Foreground stellar and background galactic contamination were assessed and found to be small. The ICMF was assumed to be equal to the mass function of clusters with ages $\leqslant 20 \mathrm{Myr}$. The same procedure was repeated on SDSS data of several nearby spiral galaxies at similar distances but with higher metallicity and shear. Several hundred clusters were identified from both the dwarf irregular and spiral galaxy samples.

The main result is that these samples are statistically indistinguishable from each other, suggesting that the ICMF does not depend on galactic shear or metallicity. We find the ICMF is reasonably well fit by a power law $\frac{d N(M)}{d M} \propto M^{-\alpha_{M}}$ with $\alpha_{M} \simeq 1.5$ in both dwarf irregular and spiral galaxies. This is somewhat shallower than the power law index of $\alpha_{M} \simeq 2$ that has been found in spiral galaxies by (Larsen 2002) using HST images. This may be due to the lower resolution of the SDSS observations, which lead to blending of clusters that form within $\sim 50 \mathrm{pc}$ of each other. Nevertheless the similarity of the cluster (or association) mass functions between the galaxy samples suggests that a universal process, perhaps turbulent fragmentation inside GMCs (Elmegreen \& Efremov 1997), is responsible for star cluster formation.

\section{Acknowledgements}

JCT acknowledges support from CLAS, University of Florida. 


\section{References}

Bally, J. \& Zinnecker, H. 2005, MNRAS 129, 2281

Beuther, H., Churchwell, E.B., McKee, C.F. \& Tan, J.C. 2006, in: B. Reipurth, D. Jewitt \& K. Keil (eds.), Protostars $\&$ Planets $V$, in press,(astro-ph/0602012)

Beuther, H. \& Schilke, P. 2004, Science 303, 1167

Beuther, H., Schilke, P., Gueth, F., McCaughrean, M., et al. 2002, A\&\&A 387, 931

Billett, O.H., Hunter, D.A. \& Elmegreen, B.G. 2002, AJ 123, 1454

Bonnell, I.A., Bate, M.R. \& Zinnecker, H. 1998, MNRAS 298, 93

Bonnell, I.A., Vine, S.G. \& Bate, M.R. 2004, MNRAS 349, 735

Caselli, P. \& Myers, P.C. 1995, ApJ 446, 665

Cesaroni, R., Galli, D., Lodato, G., Walmsley, C.M. \& Zhang, Q. 2006, in: B. Reipurth, D. Jewitt \& K. Keil (eds.), Protostars 83 Planets V, in press, (astro-ph/0603093)

Crutcher, R.M. 2005, in: R. Cesaroni, et al. (eds.), Massive star birth: A crossroads of Astrophysics (Cambridge: Cambridge Univ.), p. 98

de Wit, W.J., Testi, L., Palla, F. \& Zinnecker, H. 2005, A\& A 437, 247

Dobbs, C.L., Bonnell, I.A. \& Clark, P.C. 2005, MNRAS 360, 2

Doty, S.D., van Dishoeck, E.F. \& Tan, J.C. 2006, A\&A 454, L5

Dowell, J.D., Buckalew, B.A. \& Tan, J.C. 2007, ApJ submitted.

Edgar, R. \& Clarke, C. 2004, MNRAS 349, 678

Egan, M.P., Shipman, R., Price, S., Carey, S., Clark, F. \& Cohen, M. 1998, ApJ 494, L199

Elmegreen, B.G. 2000, ApJ 530, 277

Elmegreen, B.G. \& Efremov, Y. 1997, ApJ 480, 235

Fall, S.M., Chandar, R. \& Whitmore, B.C. 2005, ApJ 631, L133

Garmire, G., Feigelson, E.D., Broos, P., et al. 2000, AJ 120, 1426

Grosso, N., Feigelson, E.D., Getman, K.V., et al. 2005, ApJS 160, 530

Hartmann, L. \& Burkert, A. 2006, ApJ in press, (astro-ph/0609679)

Hoogerwerf, R., de Bruijne, J.H.J. \& de Zeeuw, P.T. 2001, A\& A 365, 49

Huff, E.M. \& Stahler, S.W. 2006, ApJ 644, 355

Hunter, T., Brogan, C., Megeath, S., Menten, K., et al. 2006, ApJ 649, 888

Kennicutt, R.C. 1998, ApJ 498, 541

Kratter, K. \& Matzner, C.D. 2007, MNRAS in press, (astro-ph/0609692)

Krumholz, M.R. 2006, ApJ 641, 45

Krumholz, M.R. \& McKee, C.F. 2005, ApJ 630, 250

Krumholz, M.R., McKee, C.F. \& Klein, R.I. 2005, Nature 438, 332

Krumholz, M.R., McKee, C.F. \& Klein, R.I. 2007, ApJ submitted, (astro-ph/0609798)

Lada, C.J. \& Lada, E.A. 2003, ARAA 41, 57

Larsen, S. 2002, AJ 124, 1393

Li, Z-Y. \& Nakamura, F. 2006, ApJ 640, L187

Massey, P. 1998, in: G. Gilmore, D. Howell (eds.), The Stellar Initial Mass Function (ASP-CS), 142,17

Massi, F., Testi, L. \& Vanzi, L. 2006, A\&A 448, 1007

McKee, C.F. \& Tan, J.C. 2003, ApJ 585, 850

Mueller, K.E., Shirley, Y.L., Evans, N.J. \& Jacobson, H.R. 2002, ApJS 143, 469

Reid, M.A. \& Wilson, C.D. 2006, ApJ 644, 990

Shu, F.H., Adams, F.C. \& Lizano, S. 1987, ARAA 25, 23

Shirley, Y.L., Evans, N.J., Young, K.E., Knez, C. \& Jaffe, D.T. 2003, ApJS 149, 375

Simon, R., Jackson, J.M., Clemens, D.P., Bania, T.M. \& Heyer, M.H. 2001, ApJ 551, 747

Tan, J.C. 2004, in: D. Johnstone, et al. (eds.), Star Formation in the Interstellar Medium: In Honor of David Hollenbach, Chris McKee and Frank Shu (ASP-CS), 323, 249

Tan, J.C. 2005, in: R. Cesaroni, et al. (eds.), Massive star birth: A crossroads of Astrophysics (Cambridge: Cambridge Univ.), p. 318

Tan, J.C., Krumholz, M.R. \& McKee, C.F. 2006, ApJ 641, L121

Weingartner, J.C. \& Draine, B.T. 2001, ApJ 548, 296 


\section{Discussion}

LINZ: The speaker mentioned that apparently no current star formation occurs in his sample of IRDCs. Can we be really sure about that, since in most cases, objects are found in IRDCs by Spitzer/MIPS?

TAN: There are two issues here: (1) at each location in the cloud there is a constraint on the embedded luminosity from the lack of flux at $\sim 8 \mu \mathrm{m}$, and, without having done detailed calculations, my impression is that for most of the regions of IRDCs in our sample there is no current, active, luminous star formation, i.e. massive star formation, occurring. There could be embedded lower-luminosity sources and it would be useful to probe this population (either with Spitzer/MIPS or with x-rays). (2) IRDCs are not a particularly well-defined class of objects, and there can in fact be bright sources nearby in adjacent clouds or even in part of the same cloud (the cloud in Fig. 2 has such a source). Still, if one were to measure the total light to mass ratios of IRDCs these should on the average be quite low compared to more evolved star-forming clouds.

LINZ: Still, these objects embedded there might be lower luminosity now but could develop into high-mass YSOs later on?

TAN: I agree that many or most IRDCs, especially the relatively high column density ones that we are studying, are likely to form star clusters and massive stars in the future.

FUKUI: In your turbulent picture, how could you explain the formation of super star clusters?

TAN: Observed super star clusters (SSCs) have $\sim 10^{6} M_{\odot}$ inside a sphere of radius $\sim 3$ pc. One basic open question is whether the initial condition is an essentially starless gas cloud with these properties or whether SSCs form more gradually as smaller clouds (perhaps already forming star clusters) merge with the main cluster. In my opinion, it would be difficult to produce the starless initial condition from typical Galactic GMCs without some kind of synchronized, fast trigger. The escape speed from SSCs is greater than the ionised gas sound speed, so they may be forming with very high efficiency from their parent gas clouds (requiring long formation times [and age spreads] in terms of free-fall times) (Tan \& McKee 2004, in proc. of Cancun Workshop). This longer formation time may allow more time for infall and merger of surrounding gas clouds, and the higher efficiency means less total gas mass is needed to reach the final stellar mass. 